\title{
Digitaler Stress im Smart Home: Eine empirische Untersuchung
}

\author{
Nadine Mittermüller • Thomas Fischer $\mathbb{D}$ - René Riedl $\mathbb{1}$
}

Eingegangen: 17. Januar 2019 / Angenommen: 7. März 2019 / Online publiziert: 22. März 2019

(C) Der/die Autor(en) 2019

Zusammenfassung Die Nutzung von Informations- und Kommunikationstechnologien (IKT) kann zu digitalem Stress führen. Die zunehmende Verbreitung von Smart-Home-Technologien im privaten Umfeld, die über unterschiedliche Endgeräte und Komponenten (von konventionellen Schaltern bis zu Smartphone-Apps) bedient werden können, kann die Entstehung von digitalem Stress begünstigen. Mittels einer dreistufigen methodischen Vorgehensweise wurde auf Basis der Gegenüberstellung zweier Beispiele (konventioneller Taster vs. Smartphone-App) untersucht, ob bei der Bedienung von Smart-Home-Anwendungen digitaler Stress wahrgenommen wird. Die Ergebnisse zeigen, dass eine mobile App zur Steuerung von Smart-HomeTechnologie Stress reduzierend wirken kann, und zwar insbesondere dann, wenn Erfahrung im Umgang mit der verwendeten Technologie besteht. Smart-Home-Interessenten, also Personen, welche bereits Erfahrung im Umgang mit dieser Technologie haben, jedoch nicht in einem solchen Umfeld leben, antizipieren jedoch deutlich mehr Stress als Smart-Home-Nutzer oder Personen ohne Smart-Home-Erfahrung.

Zusatzmaterial online Zusätzliche Informationen sind in der Online-Version dieses Artikels (https:// doi.org/10.1365/s40702-019-00517-6) enthalten.

\footnotetext{
N. Mittermüller $\cdot$ T. Fischer $(\bowtie) \cdot$ R. Riedl

Fakultät für Management, Abteilung für Digital Business, Fachhochschule Oberösterreich,

Wehrgrabengasse 1-3, 4400 Steyr, Österreich

E-Mail: thomas.fischer@fh-steyr.at

N. Mittermüller

E-Mail: nadine.mittermueller@gmail.com

R. Riedl

E-Mail: rene.riedl@fh-steyr.at

R. Riedl

Institut für Wirtschaftsinformatik - Information Engineering, Johannes Kepler Universität Linz, Altenberger Straße 69, 4020 Linz, Österreich
} 
Schlüsselwörter Digitaler Stress · Einstellung $\cdot$ Erfahrung $\cdot$ Interviews $\cdot$ Online Befragung · Smart Home

\title{
Digital Stress in Smart Homes: An Empirical Investigation
}

\begin{abstract}
The use of information and communication technologies (ICT) can lead to technostress. The growing prevalence of smart home technologies at home that can be controlled with a variety of devices and components (from conventional push buttons to smartphone apps) may foster the emergence of technostress. By utilizing a research design based on three steps, we investigated if technostress is experienced when controlling smart home applications through a comparison of two examples (conventional push button vs smartphone app). The results indicate that mobile apps can create a more stress-free control mode for smart homes, particularly if the individual has experience with the use of the technology. Yet, individuals who are interested in smart homes and have already gained some experience with the use of involved technologies, but do not live in a smart home, anticipate more stress than individuals who live in smart homes or individuals who have no prior experience with smart homes.
\end{abstract}

Keywords Technostress $\cdot$ Attitude $\cdot$ Experience $\cdot$ Interviews · Online Survey • Smart Home

\section{Smart-Home-Steuerung und digitaler Stress}

Bereits vor Jahrzehnten begann der Einzug von Informations- und Kommunikationstechnologien (IKT) in private Haushalte, zuerst kam der PC, dann das Internet und vor rund zehn Jahren das Smartphone. Der Einsatz von Smart-Home-Technologien (SH) gewann in den letzten Jahren an Bedeutung (Lübbeke 2016). Prognosen gehen davon aus, dass der weltweite $\mathrm{SH}-U m s a t z$ in den kommenden fünf Jahren um das 3-fache steigen wird und bis 2022 eine globale Penetrationsrate von 19,5\% erreicht sein wird (Statista 2018).

Ein SH-System besteht aus verschiedenen Komponenten, die miteinander vernetzt sind. Beispielkomponenten sind Steuerungskomponenten (z. B. Signalverteiler, Router), Sensoren und Aktoren. Kernstück von Smart-Home-Systemen ist meistens die zentrale Steuereinheit, die Signale der angeschlossenen Komponenten zusammenführt und verwaltet und über das Internet, z. B. mittels eines Cloud-Systems verbunden ist. Eine sehr einfache Beispielanwendung ist das automatische Einschalten eines Lichts. Diese basiert auf einem Sensor im Eingangsbereich einer Wohnung, der Bewegungen erfasst und infolgedessen ein Signal weitergibt, sodass das Licht im betreffenden Bereich eingeschaltet wird. Was dieses Beispiel „smart“ macht ist die Möglichkeit, dass die gleiche Konstellation eine Nachricht an das Smartphone des Wohnungsbesitzers senden kann (letzteres ist z. B. dann relevant, wenn der Besitzer nicht zu Hause ist und trotzdem Bewegung registriert wird).

Die einzelnen Funktionalitäten eines SH können dabei mit unterschiedlichen Geräten bedient werden. Die Bandbreite reicht von konventionellen Eingabegeräten 
wie Wand-Tastern oder Schaltern, über Fernbedienungen bis zu einer Eingabe via Bildschirmgeräten (z.B. Smartphones oder Tablets); zudem gewinnen Sprach- und Gestensteuerung zunehmend an Bedeutung. Befunde einer Befragungsstudie unter potenziellen Benutzern zeigen folgende Präferenzen zur Steuerung von SH-Anwendungen: $46 \%$ via Smartphone und etwa nur $12 \%$ via Notebook (Schulze-Sturm 2016).

Insbesondere die Verwendung eines Smartphones zur Steuerung kann jedoch zu zusätzlichem Bedienaufwand führen, wie etwa durch notwendiges Entsperren des Displays oder Auffinden des entsprechenden Befehls in der App. Dies schmälert den wahrgenommenen Vorteil der Nutzung einer Smartphone-App im Vergleich zur Nutzung eines herkömmlichen Tasters (Jakobi et al. 2016). Die Möglichkeit, Funktionen mit Hilfe des Smartphones von der Ferne steuern zu können, um so etwa eine Anwesenheit zu simulieren oder mit einem Befehl sämtliche Geräte ausschalten zu können, sind dagegen wesentliche Vorteile der Steuerung mittels Smartphone (Jakobi et al. 2016).

Gerade im SH-Bereich gibt es einerseits ein großes Maß an persönlicher Offenheit für neue Technologien (Venkatesh 2008), da diese eigene Verhaltensweisen besser unterstützen und das Familienleben verbessern (z. B. mehr gemeinsame Freizeit, weil die Technologie bestimmte Aufgaben übernimmt). Andererseits kann eine zu starke Digitalisierung auch Probleme mit sich bringen (z. B. Fehlfunktion in der mobilen App, welche dazu führt, dass man die Eingangstür im Smart Home nicht mehr öffnen kann). Die Allgegenwärtigkeit von und Angewiesenheit auf IKT kann negative physische und psychische Auswirkungen haben (Riedl 2013), unter anderem wird in der Fachliteratur über Schlafstörungen (Thomée et al. 2012) sowie Angstzustände (Rosen und Weil 1995) berichtet. Dieses Phänomen wird als digitaler Stress (Technostress) bezeichnet und ist bereits seit den 1980er Jahren Gegenstand der Forschung (Brod 1982), wobei die Bedeutung der Technostressforschung in den vergangenen Jahren signifikant zugenommen hat (Fischer und Riedl 2017).

Vor dem Hintergrund, dass bislang keine Forschung zu digitalem Stress im Kontext von SH-Anwendungen existiert, haben wir drei Forschungsfragen untersucht. In unserer Arbeit konzentrieren wir uns dabei - aufgrund der beschriebenen Vielfalt an Möglichkeiten im SH - auf 2 Interaktionsformen bzw. Komponenten - konventionelle Taster und mobile App.

Forschungsfrage 1 Gibt es einen signifikanten Unterschied zwischen konventionellen Tastern und einer mobilen App als Eingabemöglichkeit in Bezug auf wahrgenommenen Stress?

Stresswahrnehmungen sind jedoch nicht nur von Eigenschaften der verwendeten Komponenten abhängig, sondern können zudem von Eigenschaften der involvierten Person beeinflusst werden, wobei im Kontext von digitalem Stress die Erfahrung im Umgang mit der verwendeten Technologie relevant zu sein scheint (z.B. Riedl et al. 2013; Salanova et al. 2013) und die Einstellung gegenüber der verwendeten Technologie (z. B. Galluch et al. 2015; Ayyagari et al. 2011). Mit Fokus auf die mobile App (welche in diesem Kontext die digitale Variante der SH-Interaktion darstellt) untersuchen wir daher zudem die beiden folgenden Forschungsfragen. 
Forschungsfrage 2 Gibt es einen Unterschied zwischen Personen mit unterschiedlichem Erfahrungsgrad im Umgang mit SH-Anwendungen in Bezug auf wahrgenommenen Stress bei der Nutzung der mobilen App?

Forschungsfrage 3 Gibt es einen Unterschied zwischen Personen mit einer unterschiedlichen Einstellung gegenüber SH-Anwendungen in Bezug auf wahrgenommenen Stress bei der Nutzung der mobilen App?

\section{Empirische Studie}

Die Untersuchung der Forschungsfragen erfolgte mit einer Online-Befragung von SH-Bewohnern sowie Interessenten (z. B. Hausbesitzer, die SH-Technologie erwerben wollen, oder Personen, welche ein SH als nächsten Wohnsitz erwägen). Die Befragung mit möglichen Nutzungsszenarien bildet hierbei einen ersten, explorativen Schritt, um das Stresspotenzial unterschiedlicher SH-Steuermöglichkeiten zu ergründen. Weitere Studien, welche die tatsächliche Nutzung untersuchen (z.B. in einem Laborexperiment), sollten diese Untersuchung in Zukunft ergänzen, um etwa die Validität der Ergebnisse zu stärken. Vor der eigentlichen Befragung wurden Vorarbeiten geleistet, um die Nutzungsszenarien auswählen zu können. Dies umfasste zuerst eine Analyse der Fachliteratur, um Faktoren zu identifizieren, welche die Auswahl einer Eingabemethode (z. B. Taster, mobile App) beeinflussen. Dieser Schritt wurde durchgeführt, da es mittlerweile SH-Anbieter gibt, welche diese Auswahl ermöglichen (z. B. Loxone Taster $^{1}$ und Loxone SH app ${ }^{2}$ ). Im Anschluss wurden Interviews mit SH-Nutzern geführt, um die Liste an Faktoren zu überprüfen und Bereiche zu identifizieren, die in Bezug auf die mobile App als Eingabemethode als besonders negativ wahrgenommen werden. Die Interviewergebnisse wurden anschließend für die Entwicklung der Nutzungsszenarien herangezogen.

\subsection{Entwicklung der Nutzungsszenarien}

Im Dezember 2017 wurde zuerst eine Literaturanalyse nach Webster und Watson (2002) durchgeführt, die 16 Publikationen umfasste. Auf Basis der inhaltlichen Analyse dieser Publikationen wurden sechs wesentliche Kategorien an Einflussfaktoren identifiziert, welche die Auswahl einer Eingabemöglichkeit beeinflussen können: Art der Aufgabe, zeitlicher Aufwand, Eigenschaften der Nutzer, Bequemlichkeit und Gewohnheit, Erreichbarkeit des Gerätes, Kontrolle. Diese Kategorien bildeten im Anschluss den Rahmen für die Interviews mit SH-Bewohnern.

Im nächsten Schritt wurden im Frühjahr 2018 Interviews mit zehn SH-Bewohnern, die sowohl eine mobile App (Hersteller: Loxone ${ }^{3}$ ) sowie auch konventionelle Taster zur Steuerung verwenden (3 weiblich, 7 männlich; Durchschnittsalter: 31 Jah-

\footnotetext{
1 https://shop.loxone.com/dede/loxone-etouch.html [24.02.2019].

2 https://www.loxone.com/dede/produkte/apps/ [24.02.2019].

3 https://www.loxone.com/dede/ [24.02.2019].
} 
Tab. 1 Sentimentanalyse der mobilen App nach Kategorien

\begin{tabular}{llll}
\hline Anzahl der Codings je Kategorie & Positiv & Neutral & Negativ \\
\hline Kundensupport & $1(100 \%)$ & $0(0 \%)$ & $0(0 \%)$ \\
Kompatibilität & $2(29 \%)$ & $0(0 \%)$ & $5(71 \%)$ \\
Kosten & $6(33 \%)$ & $0(0 \%)$ & $12(67 \%)$ \\
Anpassungen/Wartung & $17(53 \%)$ & $3(9 \%)$ & $12(38 \%)$ \\
Updates & $0(0 \%)$ & $0(0 \%)$ & $4(100 \%)$ \\
Art der Aufgabe & $0(0 \%)$ & $26(100 \%)$ & $0(0 \%)$ \\
Zeitlicher Aufwand & $6(27 \%)$ & $3(14 \%)$ & $13(59 \%)$ \\
Eigenschaften der Nutzer & $1(4 \%)$ & $21(88 \%)$ & $2(8 \%)$ \\
Bequemlichkeit und Gewohnheit & $21(84 \%)$ & $0(0 \%)$ & $4(6 \%)$ \\
Erreichbarkeit des Gerätes & $1(5 \%)$ & $14(64 \%)$ & $7(31 \%)$ \\
Kontrolle & $31(76 \%)$ & $7(17 \%)$ & $3(7 \%)$ \\
Sicherheit & $2(10 \%)$ & $7(33 \%)$ & $12(67 \%)$ \\
Gesamt & $88(36 \%)$ & $81(33 \%)$ & $74(31 \%)$ \\
\hline
\end{tabular}

re; Leben im Durchschnitt seit 2 Jahren in einem SH), durchgeführt. Der Interviewleitfaden basierte auf den identifizierten Kategorien.

Die Interviewpartner wurden dazu befragt, wie die jeweiligen Steuerungsmöglichkeiten die zuvor identifizierten Kategorien unterstützen beziehungsweise wie sie diese einschränken. Zudem wurde nach möglichen weiteren die Steuerung beeinflussenden Kriterien gefragt. Es wurden fünf weitere Kategorien identifiziert: Kundensupport, Kompatibilität, Kosten, Anpassungen/Wartung und Updates.

Im Anschluss an die Transkription der Interviews wurde von der Erstautorin eine Inhaltsanalyse (Mayring 2015) mit Hilfe von MAXQDA durchgeführt, um eine Sentimentanalyse (Pang und Lee 2008) der Aussagen durchzuführen. Dabei wurden anhand der Erzählungen der Interviewpartner, Aussagen zu den beiden Technologien (Taster und mobile App) zuerst einer Kategorie zugeordnet und anschließend wurde bewertet, ob die Aussagen als neutral, positiv oder negativ eingestuft werden können. Von 243 getätigten Aussagen die den Eingabemöglichkeiten zugeordnet werden können, waren $36 \%$ positiv, $33 \%$ neutral und $31 \%$ negativ (siehe Tab. 1; der relative Anteil der Bewertungen je Kategorie ist zudem in Klammern angegeben).

Die Kategorie zeitlicher Aufwand wurde insbesondere in einem negativen Zusammenhang erwähnt. Die negative Beurteilung beruht etwa darauf, dass die Bedienung der Funktionen mit der Smartphone-App länger dauert als mit dem Taster und daher als umständlicher betrachtet wird.

In der Kategorie Anpassungen/Wartung gab es eine leichte Tendenz hin zum Positiven. Die negativen Aspekte dieser Kategorie werden häufig im Zusammenhang mit Systemeinstellungsproblemen genannt, denn diese treten erst im Laufe der Nutzung zutage. Sie zu beheben ist allerdings zeitaufwändig und erfordert ein gewisses Ausmaß an Durchhaltevermögen.

Für die Online-Befragung wurden zwei Szenarien entwickelt, die jeweils für den Fall der Nutzung der mobilen App und des konventionellen Tasters leicht variiert wurden. Szenario 1 (,Funktion“) bezieht sich auf den zeitlichen Aufwand, der mit der Nutzung der mobilen App verbunden ist und beschreibt, wie in einem Neben- 
raum das Licht ausgeschaltet werden soll, während man sich einen Film ansieht. Die Funktion dafür muss in der App mit etwas Aufwand gesucht werden und es kann dabei zu Problemen mit der Internetverbindung kommen, weshalb die Funktion erneut ausgeführt werden muss. Im Fall des konventionellen Tasters muss der Film angehalten werden und man muss zur Steuereinheit gehen, um das Licht auszuschalten. Szenario 2 (,Einstellung“) bezieht sich auf den Aufwand der Anpassung des Systems. Dabei wird man am Wochenende früh morgens von den sich automatisch öffnenden Jalousien geweckt, deren Steuerung in einem Programm im System definiert/hinterlegt ist. Diese Einstellung soll dahingehend verändert werden, dass die Öffnung am Wochenende zu einem späteren Zeitpunkt erfolgt. Im Fall der mobilen App handelt es sich um eine selten verwendete Einstellung, die zuerst einige Zeit lang gesucht werden muss und bei der noch nicht unmittelbar festgestellt werden kann, ob die Änderung der Einstellung funktioniert hat (da diese erst am nächsten Tag effektiv wird). Im Fall des konventionellen Tasters kann eine Veränderung nicht selbst vorgenommen werden, sondern es muss eine Person, welche für die Wartung zuständig ist, kontaktiert werden, um die Einstellung vorzunehmen.

\subsection{Online-Befragung}

Eine Online-Befragung wurde von 8. bis 29. Juni 2018 durchgeführt, wobei insgesamt 93 Personen teilnahmen (40\% Frauen, 60\% Männer; Durchschnittsalter: 33 Jahre), die sich aus SH-Bewohnern, Personen mit SH-Erfahrung und Personen ohne SH-Erfahrung zusammensetzten. $75 \%$ der Befragten gaben an, bereits Erfahrungen mit SH gesammelt zu haben, entweder im eigenen Zuhause, da sie in einem SH leben oder bei der Familie, Freunden oder Bekannten.

Der Stresslevel, welcher durch die beiden Szenarien induziert werden sollte, wurde durch den PASA-Fragebogen (primary appraisal, secondary appraisal) erhoben (Gaab 2009). Der PASA-Fragebogen ermöglicht die Berechnung eines Stressindex, der von -6 (kein Stress) bis +6 (sehr hoher Stress) reichen kann. Der Fragebogen setzt sich aus vier Dimensionen und insgesamt 16 Items zusammen. Die Items werden über eine siebenstufige Likert-Skala gemessen ( 1 - stimme überhaupt nicht zu bis 7 - stimme völlig zu). Die Erfahrung mit SH wurde über eine Frage mit drei Antwortmöglichkeiten (keine Erfahrung; Erfahrung, aber lebe nicht selbst in einem SH; Erfahrung und lebe selbst in einem $\mathrm{SH}$ ) erhoben. Für die Einstellung gegenüber SH wurde ein semantisches Differential mit sieben Adjektivpaaren verwendet (z. B. ,Ein Smart Home ist für mich ... schlecht/gut"), wobei sechs Stufen für die Bewertung zur Verfügung standen. (Siehe zu den verwendeten Fragebögen Onlinematerial 1).

Vor der eigentlichen Befragung wurde zudem ein Pre-Test mit drei Personen durchgeführt, um die Verständlichkeit der Fragen und den Wirklichkeitsbezug der Szenarien zu prüfen. Es handelte sich um Nutzer der untersuchten Systeme, denen die Szenarien vorgelegt wurden und die dann in eigenen Worten widergeben sollten, was das Problem in diesem Szenario darstellt. Es wurde dann besprochen, ob es sich um eine realistische Situation handelt und die Formulierung wurde angepasst, wenn nicht direkt verständlich war, um welche Art Szenario es sich hier handelt. Bei keiner der drei Personen traten hinsichtlich Verständlichkeit oder Wirklichkeitsbezug gravierende Unklarheiten bzw. Probleme auf. 


\section{Ergebnisse und Implikationen für die Praxis}

Es werden die Ergebnisse der Online-Befragung sowie deren Implikationen für die Praxis vorgestellt. Dabei werden die zuvor vorgestellten Forschungsfragen zur Gliederung herangezogen.

\subsection{Ergebnisse der Online-Befragung}

Für die Beantwortung von Forschungsfrage 1 wurden Mann-Whitney-U-Tests mit den Antworten der SH-Bewohner $(N=37)$ durchgeführt. (Da die Daten der Befragung nicht normalverteilt sind, wurden in weiterer Folge nicht-parametrische Tests angewendet.) Für Szenario 1 (Schalten des Lichts in einem Nebenraum) wurde bei der Verwendung der mobilen App mehr Stress antizipiert (Mittelwert: -2,72; St.abw.: 2,13) als mit dem konventionellen Taster (Mittelwert: -3,41; St.abw.: 1,60), wobei dieser Unterschied statistisch nicht signifikant ist $(\mathrm{U}=548,00, p=0,140)$. Für Szenario 2 wurde bei der Verwendung der mobilen App weniger Stress antizipiert (Mittelwert: $-3,40$; St.abw.: 1,58) als mit dem konventionellen Taster (Mittelwert: $-0,79$; St.abw.: 2,69), wobei dieser Unterschied statistisch signifikant ist ( $U=281,00$, $p<0,001)$. In Bezug auf Forschungsfrage 1 kann daher nicht mit Sicherheit festgestellt werden, ob die mobile App zu weniger Stress in der Verwendung führt. Da jedoch in keinem der Szenarien signifikant mehr Stress durch die App erzeugt wird und es sich um Szenarien handelt, die bewusst aufgrund ihres Stresspotenzials im Umgang mit der App ausgewählt wurden, ist eine Tendenz zu weniger Stress bei der Verwendung einer mobilen App zur Steuerung von SH-Funktionalitäten ableitbar.

Für Forschungsfrage 2 wurden die Daten aller 93 Befragten herangezogen, um den Unterschied in der Erfahrung mit SH (23 Personen ohne Erfahrung, 33 Personen mit Erfahrung, 37 SH-Bewohner) als Einflussfaktor auf den antizipierten Stress im Umgang mit der mobilen App zu untersuchen. Zudem wurden auch die Unterschiede in Bezug auf den konventionellen Taster untersucht, um einen weiteren Vergleich der Ergebnisse zu ermöglichen. Es wurde dabei zuerst eine ANOVA für alle Varianten (zwei Eingabemöglichkeiten $\times$ zwei Szenarien) berechnet, bei der die drei Gruppen als unabhängige Variable verwendet wurden (Tab. 2). Es wurden zudem die Eingabemöglichkeiten über beide Szenarien hinweg analysiert und ein globaler Stresswert über alle vier Möglichkeiten berechnet und für die drei Gruppen verglichen.

Ein erstes unerwartetes Ergebnis ist, dass sich ein nichtlinearer Zusammenhang zwischen der Erfahrung mit SH-Technologien und dem wahrgenommenen Stress in den untersuchten Szenarien abzeichnet. Wie in Tab. 2 ersichtlich, antizipieren Personen mit SH-Erfahrung, die jedoch selbst keine SH-Bewohner sind, in den meisten Fällen den größten Stress. Nur in Szenario 1 unter Verwendung der mobilen App antizipieren SH-Bewohner noch mehr Stress. In den meisten Fällen ist der Unterschied jedoch statistisch nicht signifikant. Lediglich in Szenario 2 bei Verwendung der mobilen App ergab sich ein annähernd statistisch signifikanter Unterschied zwischen den Gruppen und ein Post-hoc-Test (Dunn-Bonferroni) ergab, dass konkret ein ebenfalls annähernd signifikanter Unterschied zwischen den Befragten mit erster Erfahrung im Umgang mit SH und den SH-Bewohnern besteht $(p=0,070)$. 
Tab. 2 Unterschiede im wahrgenommenen Stress nach SH-Erfahrung

\begin{tabular}{lllll}
\hline & Keine Erfahrung & Erfahrung & SH-Bewohner & KW Test \\
\hline Szenario 1 (Taster) & $-2,97$ & $-2,89$ & $-3,41$ & $\chi^{2}=1,076$ \\
& & & & $p=0,584$ \\
Szenario 1 (App) & $-2,95$ & $-2,45$ & $-2,72$ & $\begin{array}{l}\chi^{2}=0,841 \\
p=0,657\end{array}$ \\
Szenario 2 (Taster) & $-2,10$ & $-1,85$ & $-0,79$ & $\chi^{2}=4,138$ \\
& & & & $p=0,126$ \\
Szenario 2 (App) & $-2,54$ & $-2,20$ & $-3,40$ & $\chi^{2}=5,506$ \\
& & & & $p=0,064$ \\
Taster (beide Szenarien) & $-2,96$ & $-2,67$ & $-3,07$ & $\chi^{2}=0,601$ \\
& & & & $p=0,741$ \\
App (beide Szenarien) & $-2,32$ & $-2,02$ & $-2,10$ & $\chi^{2}=0,220$ \\
& & & & $p=0,896$ \\
Globale Stresswerte & $-2,64$ & $-2,35$ & $-2,58$ & $\chi^{2}=0,185$ \\
& & & $p=0,911$ \\
\hline
\end{tabular}

In Bezug auf Forschungsfrage 2 kann daher festgestellt werden, dass Erfahrung die Stresswahrnehmung bei der Bedienung von SH-Funktionalitäten ungünstig beeinflussen kann. Ein genereller Zusammenhang über mehrere Szenarien hinweg (letzte Zeile in Tab. 2) konnte jedoch nicht nachgewiesen werden.

Für Forschungsfrage 3 wurde mit einem semantischen Differential erhoben, wie positiv oder negativ die Befragten gegenüber $\mathrm{SH}$ eingestellt sind, wobei 7 den positivsten Wert darstellt. Hier ergab sich ein klares Bild für die unterschiedlichen Erfahrungsgruppen, wobei mehr Erfahrung mit einer positiveren Einstellung gegenüber SH zusammenhängt (keine Erfahrung: 3,82; erste Erfahrung: 4,27; SHBewohner: 5,31).

Für die Untersuchung des Einflusses der Einstellung auf die Antizipation von Stress wurde die Gruppe der SH-Bewohner mit einem Median-Split weiter unterteilt, und zwar in Personen mit einer weniger positiven Einstellung gegenüber SH $(N=16$; Einstellung: 4,79) und einer sehr positiven Einstellung gegenüber SH ( $N=21$; Einstellung: 5,70). Im Anschluss wurden Mann-Whitney-U-Tests für die einzelnen Szenarien berechnet, um festzustellen, ob es einen Unterschied zwischen diesen Gruppen gibt.

In allen vier Szenarien gab es keinen statistisch signifikanten Unterschied (Szenario 1 mit Taster: $\mathrm{U}=145,50, p=0,495$; Szenario 2 mit Taster: $\mathrm{U}=185,50, p=0,591$; Szenario 1 mit App: $U=138,50, p=0,370$; Szenario 2 mit App: $U=145,50$, $p=0,495)$. In Bezug auf Forschungsfrage 3 kann daher geschlossen werden, dass die Stresswahrnehmung nicht aufgrund unterschiedlicher Einstellungen gegenüber SH variiert.

\subsection{Implikationen für die Praxis}

Venkatesh (2008) stellten vor mehr als zehn Jahren fest, dass SH-Technologien komplex sind und es daher bei den Nutzern im Zuge der Verwendung zu Verwirrungen 
kommen kann und die Nutzer ihre Kenntnisse erweitern müssen, um eine erfolgreiche Nutzung sicherstellen zu können. Diese Erkenntnis wurde an dieser Stelle für zwei Eingabemöglichkeiten untersucht, wobei die Verwendung einer mobilen App als neuere Technologie (im Vergleich zu einem Taster) herangezogen wurde.

Eine wesentliche Erkenntnis der vorliegenden Studie ist, dass die Verwendung der mobilen App keinen zusätzlichen Stress verursacht; vielmehr trägt die App in manchen Situationen sogar zur Vermeidung von Stresswahrnehmungen bei. Da es sich beim verwendeten Sample mehrheitlich um jüngere Personen (Durchschnittsalter 33 Jahre bei der Online-Befragung) in ihrem ersten Eigenheim handelt, hat dies Bedeutung für die zukünftige Bewerbung von SH. So kann eine mobile App als Steuerelement stressreduzierend wirken, insbesondere, wenn dadurch die Unabhängigkeit von Drittleistern (z.B. im Fall von Änderung von Werkseinstellungen der vernetzten Systeme wie in Szenario 2) unterstützt wird. Eine weitere Erkenntnis, die herbei von Bedeutung ist, ist der höhere Stresslevel von SH-Interessenten. Diese fühlten sich deutlich stärker von den vorgestellten Szenarien belastet als Personen ohne jegliche Erfahrung im Umgang mit SH oder SH-Bewohner. Der tatsächliche Umgang mit den involvierten Technologien, wie etwa Steuerelementen, scheint daher den wahrgenommenen Stress zu reduzieren. Daraus folgt, dass Interessenten die Möglichkeit geboten werden sollte, diese Technologien vorab zu testen und zu vergleichen. Dies ist wiederum ein Argument für die stärkere Verwendung mobiler Apps, da diese mit weniger Aufwand demonstriert werden können (z.B. über Tutorials im Internet) bzw. zumeist selbsterklärend sein sollten.

Was die Einstellung gegenüber SH betrifft, konnte im Wesentlichen kein Einfluss auf die Stresswahrnehmung festgestellt werden. Es wurde zwar vorweg angenommen, dass die mobile App eine stärkere Repräsentation des SH-Gedankens darstellt als eine konventionellere Steuereinheit und daher bei „Enthusiasten“ zu weniger Stress in der Verwendung führen könnte. Dies wurde durch die Ergebnisse der Befragung jedoch nicht bestätigt. Ein Grund hierfür könnte die ohnehin sehr positive Einstellung gegenüber SH unter SH-Bewohnern sein, die zu einem zu geringen Unterschied zwischen den Gruppen geführt hat. Weiter kann nicht ausgeschlossen werden, dass Unterschiede zwischen den Gruppen mit zunehmender Stichprobengröße signifikant werden. Aufgrund der geringen Unterschiede in absoluten Werten wären jedoch solche statistisch signifikanten Unterschiede praktisch kaum von Relevanz. Zudem kann der Einfluss weiterer Variablen, wie etwa der Risikowahrnehmung in Bezug auf SH-Technologien (Hubert et al. 2018) nicht ausgeschlossen werden.

\section{Zusammenfassung}

Durch zwei Szenarien, welche von zuvor interviewten SH-Bewohnern als besonders stressauslösend eingestuft wurden, konnte auf der Basis einer Befragungsstudie festgestellt werden, dass die Steuerung eines SH mit Hilfe einer mobilen App keinen zusätzlichen Stress auslöst. Im Gegenteil, es zeigte sich in einigen Fällen, dass die Steuerung einer Vielzahl vernetzter Technologien über das vertraute Smartphone anstelle einer zusätzlichen Technologie (Steuereinheit, welche vom Hersteller zur Verfügung gestellt wird) teilweise dabei hilft, Stress zu vermeiden. Eine weitere 
wichtige Erkenntnis ist, dass die Vertrautheit mit der verwendeten Technologie (Erfahrung im Umgang) den wahrgenommenen Stress signifikant reduzieren kann.

Diese Erkenntnis ist für Hersteller von SH-Lösungen von Bedeutung, da sich Interessenten teils stärker von herausfordernden Szenarien belastet fühlen als SHBewohner, die bereits Erfahrung im Umgang mit der Steuerung der verwendeten Technologien sammeln konnten.

Wir rufen zu weiteren Untersuchungen im Kontext digitaler Stress und SH auf, insbesondere um methodische Limitationen der vorliegenden Studie zu beheben:

- Experimentelle Forschung, die den Einfluss von Eingabemöglichkeiten auf den Stress untersucht. Hierbei sind zudem Multi-Methoden-Ansätze erstrebenswert, um die Stresswahrnehmung der Studienteilnehmer mit anderen Datenquellen wie biologischen Stressreaktionen (z.B. Riedl et al. 2012) zu triangulieren (Fischer und Riedl 2017).

- Ein Vergleich mobiler Apps auf Funktionsebene ist notwendig, um etwa die am häufigsten genutzten Funktionen auf ihr Stresspotenzial hin zu untersuchen. In der vorliegenden Untersuchung wurden bewusst stressauslösende Situationen gewählt, die jedoch nicht zwingend häufig auftretende Szenarien wiederspiegeln (z.B. Ändern des Timers bei den Jalousien).

- Eine Längsschnitt-Untersuchung ist notwendig, um die Entwicklung des Stressempfindens beim Umgang mit der mobilen App weiter zu ergründen.

Funding Open access funding provided by University of Applied Sciences Upper Austria.

Open Access Dieser Artikel wird unter der Creative Commons Namensnennung 4.0 International Lizenz (http://creativecommons.org/licenses/by/4.0/deed.de) veröffentlicht, welche die Nutzung, Vervielfältigung, Bearbeitung, Verbreitung und Wiedergabe in jeglichem Medium und Format erlaubt, sofern Sie den/die ursprünglichen Autor(en) und die Quelle ordnungsgemäß nennen, einen Link zur Creative Commons Lizenz beifügen und angeben, ob Änderungen vorgenommen wurden.

\section{Literatur}

Ayyagari R, Grover V, Purvis R (2011) Technostress: technological antecedents and implications. MIS Q 35:831-858

Brod C (1982) Managing technostress: optimizing the use of computer technology. Pers J 61:753-757

Fischer T, Riedl R (2017) Technostress research: a nurturing ground for measurement pluralism? Commun Assoc Inf Syst 40:375-401

Gaab J (2009) PASA—primary appraisal secondary appraisal. Verhaltenstherapie 19:114-115. https://doi. org/10.1159/000223610

Galluch P, Grover V, Thatcher JB (2015) Interrupting the workplace: examining stressors in an information technology context. J Assoc Inf Syst 16:1-47

Hubert M, Blut M, Brock C, Zhang RW, Koch V, Riedl R (2018) The influence of acceptance and adoption drivers on smart home usage. Eur J Mark 6:181. https://doi.org/10.1108/EJM-12-2016-0794

Jakobi T, Ogonowski C, Castelli N, Stevens G, Wulf V (2016) Smart Home Experience Journey: Über den Einsatz und die Wahrnehmung von Smart Home-Technologien im Alltag. Mittelstand Digit Wiss Trifft Prax 4:12-20

Lübbeke T (2016) Akzeptanz von Smart Homes - von der Theorie zur Vermarktung. Mittelstand Digit Wiss Trifft Prax 4:21-26

Mayring P (2015) Qualitative Inhaltsanalyse; Grundlagen und Techniken. Beltz, Weinheim, Basel

Pang B, Lee L (2008) Opinion mining and sentiment analysis. Foundations and trends ${ }^{\circledR}$ in. Inf Retr Boston 2:1-135. https://doi.org/10.1561/1500000011 
Riedl R (2013) On the biology of technostress: literature review and research agenda. Data Base Adv Inf Syst 44:18-55

Riedl R, Kindermann H, Auinger A, Javor A (2012) Technostress from a neurobiological perspective system breakdown increases the stress hormone cortisol in computer users. Bus Inf Syst Eng 4:61-69

Riedl R, Kindermann H, Auinger A, Javor A (2013) Computer breakdown as a stress factor during task completion under time pressure: identifying gender differences based on skin conductance. Adv Hum Comput Interact:1-8. https://doi.org/10.1155/2013/420169

Rosen LD, Weil MM (1995) Computer anxiety: a cross-cultural comparison of university students in ten countries. Comput Hum Behav 11:45-64. https://doi.org/10.1016/0747-5632(94)00021-9

Salanova M, Llorens S, Cifre E (2013) The dark side of technologies: technostress among users of information and communication technologies. Int J Psychol 48:422-436

Schulze-Sturm S (2016) Blickwinkel Smart Home - Studien aus Angebots- und Nachfragesicht. Mittelstand Digit Wiss Trifft Prax 4:5-11

Statista (2018) Smart Home; weltweit. https://de.statista.com/outlook/279/100/smart-home/weltweit. Zugegriffen: 7. März 2019

Thomée S, Härenstam A, Hagberg M (2012) Computer use and stress, sleep disturbances, and symptoms of depression among young adults - a prospective cohort study. BMC Psychiatry 12:176

Venkatesh A (2008) Digital home technologies and transformation of households. Inf Syst Front 10:391-395. https://doi.org/10.1007/s10796-008-9097-0

Webster J, Watson RT (2002) Analyzing the past to prepare for the future: writing a literature review. MIS Q 26:xiii-xxiii 